\title{
SYNTHESIS OF Y- NI ALLOY BY CALCIOTHERMIC REDUCTION DIFFUSION PROCESS
}

\author{
Marimuthu Ilayaraja ${ }^{1}$, L. John Berchmans ${ }^{2}$, Sankara Raman Sankaranarayanan ${ }^{1 *}$ \\ ${ }^{1}$ Department of Metallurgical and Materials Engineering \\ National Institute of Technology, Tiruchirappalli - 620 015, Tamilnadu, India \\ ${ }^{2}$ Principal Scientist, Electro pyro metallurgy division, CSIR - CECRI, \\ Karaikudi, Tamilnadu, India
}

Received 19.08.2014

Accepted 26.03.2015

\begin{abstract}
In this study, magnetic material of the Yttrium based alloy such as nickel intermetallic compound is prepared by calciothermic reduction process (CRD), at different temperatures, for 7 hours, under Argon atmosphere. Kinetic analyses have been used to calculate the conversion rate and the rate constant at different temperatures. Thermodynamic calculations have been performed to estimate the Gibbs free energy at different temperatures. Scanning Electron Microscopy (SEM), X-ray diffraction and EDX analysis have been performed to characterize the samples produced at $1273 \mathrm{~K}$. Magnetic properties have been estimated using Vibrating Sample Magnetometer.

Key words: Calciothermic Reduction Diffusion process (CRD), intermetallics, unreacted core model theory, Gibbs free energy, VSM technique

\section{Introduction}

Recently, we have been working on a relatively novel route to prepare rare earth and transition metal alloys by calciothermic reduction diffusion (CRD) process. Rare earth and transition metal alloys provide many potential applications in industry. Among many others, applications as permanent magnets and magneto -optical storage materials are significant. At present, these alloys are mainly prepared by smelting at high temperature and by mechanical alloying. One common feature of these methods is that the starting materials employed are high purity metals. This consumes, unnecessarily, additional energy and increased the production cost, was discussed [1]. Permanent magnet materials are of growing interest to the electronics industry and to
\end{abstract}

\footnotetext{
*Corresponding author: Sankara Raman Sankaranarayanan, raman@nitt.edu
} 
the manufacturers of electro-mechanical devices in many electronic apparatus, circuits, electric motors and defence products [2].

In many instances of commercial importance an alloy, rather than a metal, is obtained as the product of calciothermic reduction. The alloy may be an intermediate in metal preparation or even the end product of the process. Calciothermic reduction of samarium oxide, in the presence of cobalt powder, yields samarium-cobalt alloys in the powder form. The process is popularly known as reduction diffusion. The reduction diffusion process has also been used for the production of powders of the magnetic neodymium-iron-boron alloy $\left(\mathrm{Nd}_{15} \mathrm{Fe}_{77} \mathrm{~B}_{8}\right)$. The reaction involves use of a powder mix of neodymium oxide, iron, ferroboron and calcium. This presents no difficulty because calcium can easily reduce rare earth oxide. The yield of alloy in such processes ranges from $97 \%$ to $99 \%$. Reduction diffusion is the method by which most of the 500 to 600 Tonnes of the magnetic samarium-cobalt alloy $\left(\mathrm{SmCo}_{5}\right)$ are produced every year [3]. Several other useful modifications of calciothermic reduction have been successfully developed for the preparation of this neodymium-bearing magnetic alloy [3]. The Y-Co alloy system has been studied and reported earlier [4]. The presence of rare-earth metals in magnet alloys has a surprising influence on magnetic properties, and hence the measurement of magnetic properties is much important, in the context of developing new magnetic materials. This new generation of lanthanide - based intermetallic materials has excellent magnetic properties [5]. The calciothermic reduction technique presented here has obvious advantages over the others, with rare earth oxide as raw material (which is cheaper than metallic RE) used in the melting process, and produces the alloy in powder form [6]. In the present work, we attempt the preparation of $\mathrm{YNi}_{2}$ from the yttrium oxide, metallic nickel powder with calcium as the reductant.

\section{Materials and Methods}

Yttrium oxide $\left(\mathrm{Y}_{2} \mathrm{O}_{3}\right)$, metallic Nickel powder and Calcium granules are taken in stoichiometric proportions. These powders are mixed roughly and then ground with mortar, for 15 minutes. These mixtures are made into the pellets of dimensions of $30 \mathrm{~mm}$ diameter and $10 \mathrm{~mm}$ length, using hydraulic press, with applied as load of 1.44 tonne $/ \mathrm{cm}^{2}$. The pellet is kept in the high density alumina crucible having dimensions of $50 \mathrm{~mm}$ diameter and $70 \mathrm{~mm}$ length. The crucible is kept in the high temperature furnace, at temperature of $1273 \mathrm{~K}$, under Argon pressure, for 7 hours. Afterwards, the pellets are taken from the furnace, washed with $5 \%$ acetic acid and subsequently with distilled water. The $\mathrm{CaO}$ is removed by the washed with distilled water, then the useful product is taken, for detailed analysis.

\section{Characterization}

X-ray powder diffraction patterns were recorded with a PW3040/60 X'pert PR PAN alytical, Netherlands diffractometer with $\mathrm{Cu} \mathrm{K}_{\mathrm{a}}$ radiation. The morphology was examined with an instrument of Hitachi, Japan scanning electron microscope. Chemical composition of the samples was examined by Energy dispersive-X-ray analyzer. The mass change and heat of evolution are examined by TG/DSC NETZSCH STA 449F3. Magnetic properties are examined by VSMModel: 7404 . 


\section{Thermodynamics of the system}

The Y-Ni binary system has been studied extensively. Even though, there is no data of formation free energy of Y-Ni which could be used [7].

In the $\mathrm{CRD}$ process preparation, the following reaction was considered:

$\mathrm{Y}_{2} \mathrm{O}_{3}(\mathrm{~s})+3 \mathrm{Ca}(\mathrm{g})+4 \mathrm{Ni}(\mathrm{s})=3 \mathrm{CaO}(\mathrm{s})+2 \mathrm{YNi}_{2}(\mathrm{~s})$

According to the Hess law of heat summation, equation (1) can be written as $\mathrm{Y}(\mathrm{s})+2 \mathrm{Ni}(\mathrm{s})=\mathrm{YNi}_{2}$

$\mathrm{Y}_{2} \mathrm{O}_{3}(\mathrm{~s})+3 \mathrm{Ca}(\mathrm{l})=2 \mathrm{Y}(\mathrm{s})+3 \mathrm{CaO}(\mathrm{s})$

$\mathrm{Ca}_{(1)}=\mathrm{Ca}(\mathrm{g})$

Assuming the $\mathrm{YNi}_{2}$ is an ideal solution, the entropy change of $\mathrm{YNi}_{2}$ formation at $298 \mathrm{~K}$ could be written as

$\Delta \mathrm{S}_{298}^{\mathrm{o}}=-\mathrm{R}\left(\mathrm{X}_{\mathrm{A}} \ln \mathrm{a}_{\mathrm{A}}+\mathrm{X}_{\mathrm{B}} \ln \mathrm{a}_{\mathrm{B}}\right)$

From equation (2),

$\Delta \mathrm{S}_{298}^{\mathrm{o}}=5.3217 \mathrm{JK}^{-1} \mathrm{~mol}^{-1}$

Assuming that the thermal heat capacity of $\mathrm{YNi}_{2}$ obeys the Newmann-Kopp's rule, the $\Delta \mathrm{Cp}$ of equation (2) is zero and the enthalpy change of equation (2) at $298 \mathrm{~K}$ becomes $23,452 \mathrm{~J} /$ mole.

The formation free energy of $\mathrm{YNi}_{2}$ could be obtained from equation (2)

$\Delta \mathrm{G}^{\mathrm{o}}{ }_{298(2)}=23,452.6-5.3217 \mathrm{~T}$

Using given data, equation (3),

$\Delta \mathrm{S}^{\mathbf{o}}{ }_{298(3)}=\left\{2 \mathrm{~S}_{\mathrm{Y}}^{\mathrm{o}}+3^{\circ} \mathrm{CaO}\right\}-\left\{\mathrm{S}_{\mathrm{Y} 2 \mathrm{O} 3}^{\mathrm{o}}\right\}$

$\Delta \mathrm{S}_{298(3)}^{\mathrm{o}}=104.02 \mathrm{JK}^{-1} \mathrm{~mol}^{-1}$

The enthalpy change of formation at $298 \mathrm{~K}$ in equation (3),

$\Delta \mathrm{H}^{\mathrm{o}}{ }_{298}=\left(\Delta \mathrm{H}_{\text {products }}^{\mathrm{o}}-\Delta \mathrm{H}_{\text {reactants }}^{\mathrm{o}}\right)$

$\Delta \mathrm{H}^{\mathrm{o}}{ }_{298}=-269450 \mathrm{~J} /$ mole

The Gibbs energy formation of equation (3) at $298 \mathrm{~K}$ is,

$\Delta \mathrm{G}_{298(3)}^{\mathrm{o}}=-269450-104.02 \mathrm{~T}$

From equation (4), the Gibbs energy formation at $298 \mathrm{~K}$ can be written as [7],

$\Delta \mathrm{G}^{\mathrm{o}}{ }_{298(4)}=117800-154.9 \mathrm{~T}$

Rearranging the equations (7), (12) and (13), the Gibbs energy formation of a reaction at $298 \mathrm{~K}$ is

$\Delta \mathrm{G}_{298(1)}^{\mathrm{o}}=-104744.8-269.563 \mathrm{~T}$ 
The free energy change of reaction at $1223 \mathrm{~K}, 1273 \mathrm{~K}$ and $1323 \mathrm{~K}$ could be calculated as [4],

$$
\begin{aligned}
& \Delta \mathrm{G}=\Delta \mathrm{G}_{298(1)}^{\mathrm{o}}+\mathrm{RT} \ln \mathrm{K} \\
& \Delta \mathrm{G}_{\mathrm{T}}=-104744.8-154.9 \mathrm{~T}+\mathrm{RT} \ln \left(\mathrm{P}_{\mathrm{ca}} / \mathrm{P}^{\mathrm{o}}\right)^{-3}
\end{aligned}
$$

Where the $P_{\mathrm{ca}}$ is the vapour pressure of calcium and $P^{o}$ is the standard atmospheric pressure $(101325 \mathrm{~Pa})$.

$\log P_{\mathrm{ca}}=-8.92 \times 10^{3} \mathrm{~T}^{-1}-139 \log \mathrm{T}+11.58 \mathrm{kPa}[5]$

The values of $\Delta \mathrm{G}_{1}$ at $1223 \mathrm{~K}, 1273 \mathrm{~K}$, and $1323 \mathrm{~K}$ are $-367.08 \mathrm{KJ} / \mathrm{mole}$, $-377.81 \mathrm{KJ} /$ mole, $-388.538 \mathrm{~kJ} /$ mole respectively, to produce the product $\mathrm{YNi}_{2}$.

\section{Results and Discussion}

\section{Kinetic Analysis}

The reaction of rare earth oxides and transition metal with reductant calcium granules, under a category of heterogeneous non-catalytic solid -fluid reactions (fluid being either gas or liquid), is considered. Several models for such reactions have been introduced in the past thirty years. An overview of the most practical reactions models is given in the work by Dziewinski [8]. The most commonly known and quoted model, the unreacted shrinking core model theory (USCM), was first introduced by Yagi and Kuni in 1955, and it is well described in Octave Levenspiel's Chemical reaction Engineering. The USCM is popular for its ease of application, clear physic-chemical interpretation of the phenomena it describes, and good predictability of many reacting systems. The model assumes that the reaction between fluid and solid takes place on a surface of the reacted core of solid particle and that the fluid reagents must diffuse through the external layer of the gas film and through the porous structure of the reaction product, which forms around the core [9]. Depending on the reaction steps film diffusion, internal diffusion, or surface reaction - may be slower than the others and it controls the reaction rate. It is necessary to know the rate - controlling step, for further calculations [10].

\section{Mechanism of reaction}

The chemical reaction can be analyzed by the equation (1). In $\mathrm{Ca}-\mathrm{Y}_{2} \mathrm{O}_{3}-\mathrm{Ni}$ reaction system, $\mathrm{Y}_{2} \mathrm{O}_{3}$ can be reduced by $\mathrm{Ca}$ and then $\mathrm{Y}$ which finally reacts with $\mathrm{Ni}$, and forms $\mathrm{YNi}_{2}$. The process of equation (1) consists of two steps: Under reaction temperature $\mathrm{Ca}$ vapour quickly diffuses to the surface of $\mathrm{Y}_{2} \mathrm{O}_{3}$ granules in porous specimen, where $\mathrm{Y}_{2} \mathrm{O}_{3}$ is reduced by $\mathrm{Ca}$ and then fresh $\mathrm{Y}$ is produced. The $\mathrm{Y}$ produced diffuses to the adjacent surface of Ni powder, where $\mathrm{Y}$ reacts with $\mathrm{Ni}$ to form $\mathrm{YNi}_{2}$ and its nucleus grows at the interface, then the $\mathrm{YNi}_{2}$ layer is formed gradually. The mode of $\mathrm{Y}$ vapour diffusing is by gas phase. There are two ways for $\mathrm{Y}$ to transfer on the surface of Ni powder. The reaction continues from the exterior to interior, so it can be described by unreacted-core model theory. The equation derived from the model was used to express the relation between conversion rate and time,

$$
k t=1-3(1-\alpha)^{2 / 3}+2(1-\alpha)
$$


Conversion rate $(\alpha)$ can be calculated by this equation

Conversion rate or rate of Reduction - Diffusion

$$
(\alpha)=\mathrm{My} / \mathrm{My} \times 100 \%
$$

where $\mathrm{M}_{\mathrm{Y}}$ is the weight of $\mathrm{Y}$ in $\mathrm{YNi}_{2}$ formed by the diffusion of $\mathrm{Y}$ into $\mathrm{Ni}$ and $\mathrm{M}^{\circ}{ }_{\mathrm{Y}}$ is the weight of $\mathrm{Y}$ in the raw materials.

Conversion rate was obtained experimentally, (My) weight of $\mathrm{Y}$ in raw materials is $27.55 \mathrm{~g}$ before process of $\mathrm{Y}_{2} \mathrm{O}_{3}$ and (My) weight of $\mathrm{Y}$ in $\mathrm{YNi}_{2}$ formed by diffusion of $\mathrm{Y}$ into $\mathrm{Ni}$ after process of mixed powders at different temperature are $12.07 \mathrm{~g}$ at $1223 \mathrm{~K}$, $12.72 \mathrm{~g}$ at $1273 \mathrm{~K}$ and $13.75 \mathrm{~g}$ at $1323 \mathrm{~K}$ respectively.

The reaction is controlled by resultant layer internal diffusion, as is applicable in unreacted core model theory is calculated by (18). Calculating the conversion rates at different temperatures in equation (19), we get values of $\alpha_{1}=0.44$ at $1223 \mathrm{~K}, \alpha_{2}=0.46$ at $1273 \mathrm{~K}, \alpha_{3}=0.50$ at $1323 \mathrm{~K}$. Using the values of conversion rate, we calculate the rate constant in equation (18), $\mathrm{k}_{1}=3.083 \times 10^{-6} \mathrm{sec}^{-1}, \mathrm{k}_{2}=3.59 \times 10^{-6} \mathrm{sec}^{-1}, \mathrm{k}_{3}=4.174$ $\times 10^{-6} \mathrm{sec}^{-1}$ respectively.

\section{TG/DCS analysis}

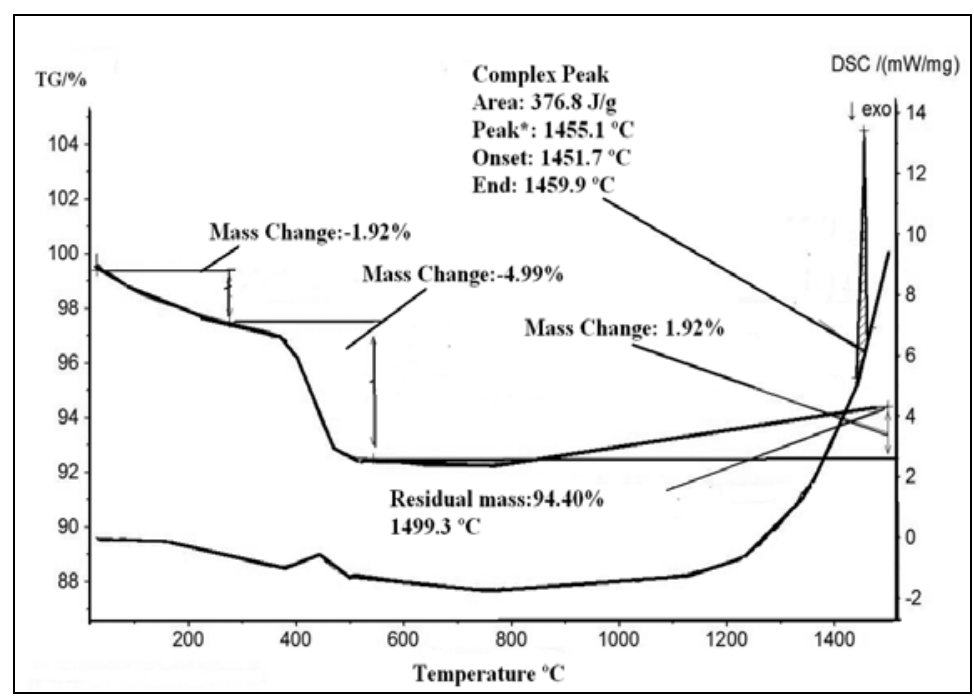

Fig. 1. Schematic digram of TG/DSC analysi of Y-Ni system produced

When the stoichiometric ratios of raw materials of Yttrium oxide, nickel powder and calcium metal were used, as presented in Figure 1., TG result reveal mass decrease for $-1.92 \%$ for the temperatures between the $100-300{ }^{\circ} \mathrm{C}$, following sudden mass decrease for $4.98 \%$ between $350^{\circ} \mathrm{C}-450^{\circ} \mathrm{C}$. From the temperature of $450^{\circ} \mathrm{C}$ the mass is relatively constant and then slightly increases for $1.92 \%$ between $1400{ }^{\circ} \mathrm{C}-1499.3{ }^{\circ} \mathrm{C}$. From the TG results it may be concluded that the mass changes in reactants, is consequence of decomposition in temperature range $100-300^{\circ} \mathrm{C}$ and $350-450^{\circ} \mathrm{C}$ and then oxidation in temperature range $1400{ }^{\circ} \mathrm{C}-1499{ }^{\circ} \mathrm{C}$. DSC result show that the start temperature is $1451.7^{\circ} \mathrm{C}$ and end temperature $1459.9^{\circ} \mathrm{C}$. The evolution of heat (enthalpy 
of reaction) shows under area $376.8 \mathrm{~J} / \mathrm{g}$, an exothermic reaction taking place at $1455.1^{\circ} \mathrm{C}$ at operating conditions at room temperature to $1500^{\circ} \mathrm{C}$ under the nitrogen atmosphere, with heating rate $10 \mathrm{~K} / \mathrm{min}$.

$X R D$ analysis

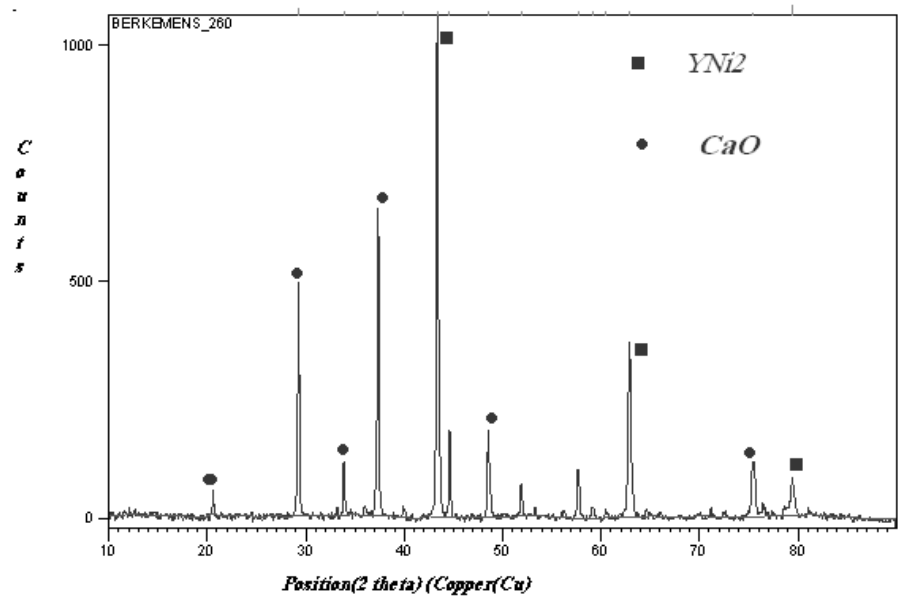

Figure 2. XRD of Y-Ni system produced at $1273 \mathrm{~K}$

Fig. 2 shows that high peaks of $\mathrm{YNi}_{2}$ and $\mathrm{CaO}$ are observed in this system.

SEM /EDX Studies

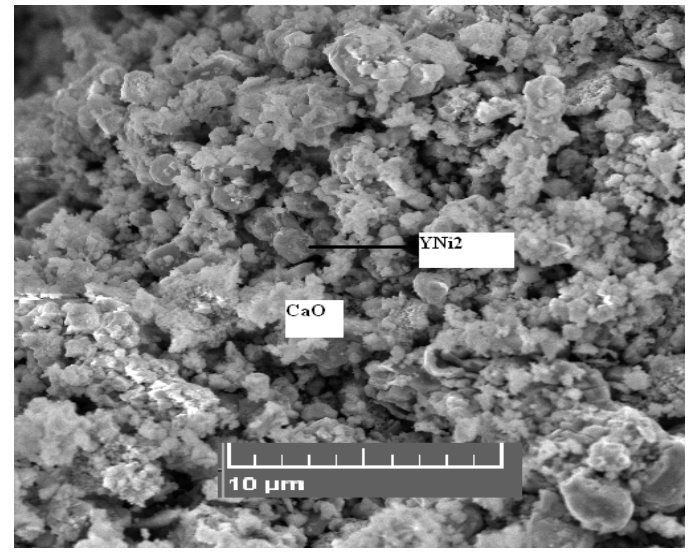

Fig. 3. (SEM) Morphology of Y-Ni system produced at $1273 \mathrm{~K}$

Figure 3 shows the scanning electron micrograph for the YNi alloy powders obtained by CRD process at $1273 \mathrm{~K}$. The SEM micrographs have shown assorted crystal features with non uniform particle morphology. The powders are crystalline in nature with fine grain size. At higher magnifications, the picture reveals the fact that the particles are 
dissociated with each other exhibiting hexagonal shape. The average particle size is measured in the range from $2 \mu \mathrm{m}-10 \mu \mathrm{m}$. The result analyzed with EDX shows that the atomic percentages of $\mathrm{Y}$ and $\mathrm{Ni}$ in $\mathrm{YNi}_{2}$ were $\mathrm{Y}=20 \%, \mathrm{Ni}=70 \%$, and the remaining $10 \%$ of $\mathrm{Ca}$ and $\mathrm{O}$ are present.

\section{VSM anaylsis}

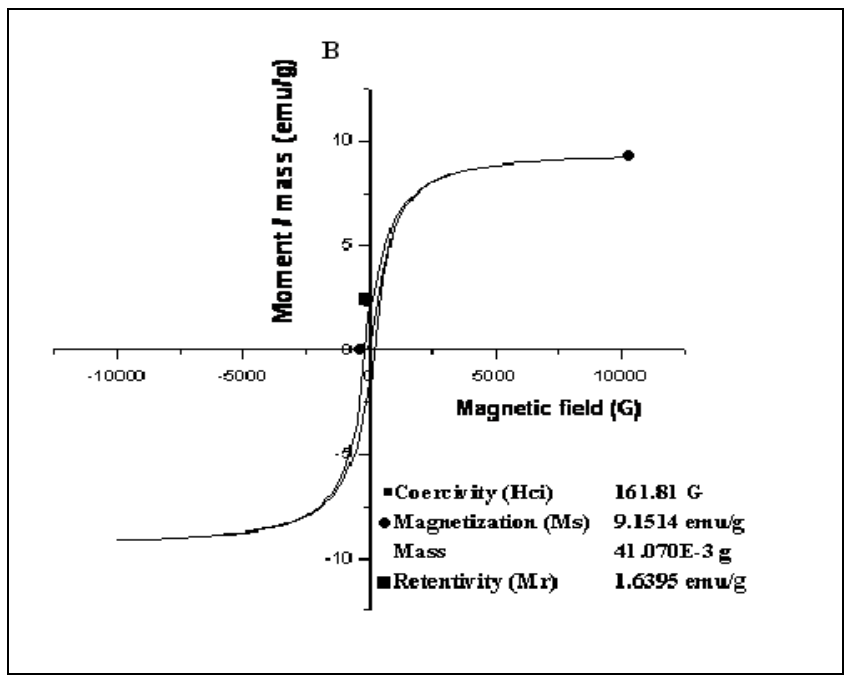

Fig. 4. (VSM) Hystersis loop of Y-Ni system produced at $1273 \mathrm{~K}$

From Figure 4, hysteresis loop described that the magnetization value is 9.1514 emu/g under plot between the magnetic field $(\mathrm{G})$ and moment/mass (emu/g) (B). The magnetic curve starts from zero with increasing magnetic field $(\mathrm{G})$ and also moment $/$ mass B $(\mathrm{emu} / \mathrm{g})$. If the magnetization curve after saturation, with applied magnetic field in opposite direction to that of the original moment/mass (emu $/ \mathrm{g})$ is known as coercivity. The coercivity value is $161.81 \mathrm{G}\left(161.81 \times 10^{-4} \mathrm{~T}\right)$. It reveals soft magnetic material must have initial permeability and a low coercivity with a relatively low applied field (i.e easily magnetized and demagnetized) and still has low hysteresis energy losses. The retentivity is used for remanence measured in units of magnetic flux density. In other words, it is the ability of a material to retain a certain amount of residual magnetic field when the magnetizing force is removed after achieving saturation. The retentivity value is $1.6395 \mathrm{emu} / \mathrm{g}$.

\section{Conclusions}

Calciothermic Reduction Diffusion process is one of the simplest techniques used to prepare pure and Intermetallic compounds. The rate constant could be calculated by unreacted core model theory. The Gibbs free energy of formation of solution at $1223 \mathrm{~K}$, $1273 \mathrm{~K}$, and $1323 \mathrm{~K}$ are $-367.08 \mathrm{KJ} / \mathrm{mole},-377.81 \mathrm{KJ} / \mathrm{mole},-388.538 \mathrm{KJ} / \mathrm{mole}$ respectively, to produce the product $\mathrm{YNi}_{2}$ (mole). The result analyzed in the EDX shows that the atomic percentages of $\mathrm{Y}$ and $\mathrm{Ni}$ in $\mathrm{YNi}_{2}$ were $\mathrm{Y}=20 \%, \mathrm{Ni}=70 \%$, respectively. The SEM image reveals that the $\mathrm{YNi}_{2}$ alloy particles are non uniform in size and shape. The SEM images indicate that the particles are in the hexagonal morphology. The average particle size is measured in the ranging from $2 \mu \mathrm{m}$ to $10 \mu \mathrm{m}$. 
From the XRD result, high peaks of $\mathrm{YNi}_{2}$ alloy and minor impurity of $\mathrm{CaO}$ are observed in this system. The grain size varies from $0.1 \mu \mathrm{m}-0.46 \mu \mathrm{m}$. The Magnetic properties of coercivity, retentivity, and magnetization values are $161.81 \mathrm{G}\left(161.81 \times 10^{-4} \mathrm{~T}\right)$, $1.6395 \mathrm{emu} / \mathrm{g}, 9.1514 \mathrm{emu} / \mathrm{g}$ respectively, as found by vibrating sample magnetometer. It is concluded that this material is a soft magnetic material.

\section{Acknowledgement}

We sincerely thank the Director National Institute of Technology Tiruchirappalli and the MHRD, Government of India, for enabling the research scholar (Mr Ilayaraja) to pursue cited research work, and for providing stipend to the scholar. We also acknowledge the Director of CSIR - CECRI for the MoU between NIT Tiruchirappalli and CSIR - CECRI, and for facilitating cited joint research. The authors are very grateful to the reviewers and the editorial team, for enabling refinement of this manuscript.

\section{References}

[1] Jianhua Lin, Shengfeng Liu, Xiaoliang Qian, JiumaBayi, Mianzeng Su, Synthesis of $\mathrm{Nd} 2 \mathrm{Fe} 17$ by Reduction-diffusion process, Journal of Alloys and compounds, vol238 , (1996), 113-115.

[2] Rare Earth Industries: Moving Malaysia's Green Economy Forward, A Report by The Academy of Sciences Malaysia \&The National Professors' Council, August 2011.

[3] Chiranjib Kumar Gupta, Chemical Metallurgy: Principles and Practice.WILEY$\operatorname{VCH}(2003), 384$.

[4] M. Ilayaraja, L. John Berchmans, Sankara Raman Sankaranarayanan, J. of Metallurgical and Materials Engineering, vol. 20, n 1, (2014), 35 - 40.

[5] T. Tanabe, Y. Nagai, T. Kubota and Z. Asaki, Mater. Transactions, JIM, 33, n 12, (1992), $1163-1170$.

[6] Verma, R.K. Sidhu, S. Mahajan, O.P. Pandey, J. of Mater. Science Letters, 15, (1996), 2088-2090.

[7] GuangsiGuo, Guangqiang Li, and Liang Liu, J. of Mater. Sci. Technol., 16, n 2, (2000), 181- 182.

[8] Journal could be found at http://www.wmsym.org/archives/1994/V2/13.pdf.

[9] O. Levenspiel, Chemical Reaction Engineering, Wiley, NY, 1972, pp. 357-400.

[10] R. O. Ajemba and O. D. Onukwuli, Am. J. Sci. Ind. Res. 3, n3, (2012), 115-121.

[11] GuangsiGuo, Guangtai Wang, and Zhitong Sui, J. of Mater. Sci. Technol., 20, n1, (2004), 68-70. 